\title{
Regulation of Carbon and Nitrogen Flow by Glutamate Synthase in Neurospora crassa
}

\author{
By YOLANDA MORA, GEORGINA HERNÁNDEZ AND JAIME MORA* \\ Centro de Investigación sobre Fijación de Nitrógeno, Universidad Nacional Autónoma de México, \\ Apartado Postal 565-A, Cuernavaca, Morelos, México
}

(Received 12 May 1986; revised 16 November 1986)

\begin{abstract}
A glycine-resistant Neurospora crassa mutant (am-132;glyr), derived from the am-132 mutant, was isolated and characterized. [am-132 itself has a deletion in the structural gene for NADPdependent glutamate dehydrogenase (GDH).] This new mutation also conferred resistance to serine and methionine sulphoximine (MS), which are inhibitors of glutamine synthetase (GS). In addition, the mutant obtained grew better on ammonium than the am-132 parental strain. Resistance to glycine was not due to increased synthesis of glutamine by an altered or induced GS, nor to increased glutamate synthesis by induction of the catabolic NAD-dependent GDH, nor to NADH-dependent glutamate synthase (GOGAT), which was as sensitive to inhibitors as the GOGAT from the parental strain. The glycine-resistance mutation lowered but did not abolish the carbon flow; this resulted in a lower content of tricarboxylic acid cycle intermediates. GOGAT activity was inhibited in vitro by several organic acids and methionine sulphone (MSF). The higher growth rate of the glycine-resistant mutant on ammonium or on ammonium plus glycine, serine or MS was explained by an increased capacity of GOGAT to synthesize glutamate in vivo due to a lower content of inhibitory tricarboxylic acid cycle intermediates; the higher glutamate content overcomes the effect of the GS inhibitors and explains the MSF resistance of the mutant.
\end{abstract}

\section{INTRODUCTION}

The regulation of nitrogen metabolism in Neurospora crassa has been investigated previously (Hummelt \& Mora, 1980a, b; Hernández et al., 1983: Lara et al., 1982; Vichido et al., 1978). The growth on ammonium of a $N$. crassa mutant that lacks NADP-dependent glutamate dehydrogenase (GDH) (EC 1.4.1.4) is inhibited by the amino acids glycine and serine (Fincham, 1950). This is due to the inhibition of glutamine synthetase (GS) (EC 6.3.1.2) which results in the diminution of the carbon flow (Hernández, et al., 1986). When nitrogen assimilation is blocked by inhibition of glutamine synthesis, carbon catabolism from sucrose decreases (Hernández \& Mora, 1986). We proposed that the drain of ATP through glutamine synthesis and the regulation of carbon metabolism by the energy charge could be considered as a point of interaction in the coordinated regulation of carbon and nitrogen metabolism.

On this basis, we now report the isolation and characterization of a glycine-resistant ( $\left.g l y^{r}\right)$ mutant strain of $N$. crassa, that has altered carbon and nitrogen flows.

\section{METHODS}

Strain. The Neurospora crassa wild-type strain $74 \mathrm{~A}$ was from the Fungal Genetics Stock Center (Humboldt State University Foundation, Arcata, Calif., USA). The mutant strain $a m-132 a$, which has a deletion in the NADPdependent GDH structural gene (Kinsey \& Hung, 1981), was kindly provided by J. A. Kinsey, Department of Microbiology, University of Kansas Medical Center, Kansas 66103, USA.

Abbreviations: GDH, glutamate dehydrogenase; GOGAT, glutamate synthase; GS, glutamine synthetase; MS, methionine sulphoximine; MSF, methionine sulphone. 
Growth conditions. Batch cultures of $N$. crassa were grown at $37^{\circ} \mathrm{C}$ on Vogel's minimal medium (N) (Vogel, 1964) containing $1.5 \%(\mathrm{w} / \mathrm{v})$ sucrose. Other nitrogen sources used in addition to ammonium nitrate are stated in the text. Growth was measured as total protein (determined by the Lowry method) as described previously (Lara et al., 1982; Vichido et al., 1978).

Mutagenesis and mutant selection. A conidial suspension of strain am-132a was prepared and adjusted to $4 \times 10^{6}$ conidia $\mathrm{ml}^{-1}$ in sterile water. A sample of the suspension $(5 \mathrm{ml})$ was placed in a Petri dish and exposed to direct UV radiation for $60 \mathrm{~s}$. After the mutagenic treatment, $0.2 \mathrm{ml}$ of the suspension were plated on $\mathrm{N}$ agar medium (Vogel, 1964) supplemented with glucose and fructose $(0.05 \%$, w/v, each), $2 \%(w / v)$ sorbose and $25 \mathrm{mm-glycine.} \mathrm{Plates}$ were incubated in the dark at $29^{\circ} \mathrm{C}$ until colonies appeared ( 2 to $7 \mathrm{~d}$ ), and then transferred to slants of $\mathrm{N}$ agar medium supplemented with $1.5 \%(\mathrm{w} / \mathrm{v})$ sucrose, and $25 \mathrm{~mm}$-glycine. Crosses were made in Corn meal agar $\left(34 \mathrm{~g}^{-1}\right)$ (Difco). Spot testing and progeny analysis were done as described by Davis \& De Serres (1970).

Determination of enzyme activities. This was done in cell-free extracts of $N$ crassa prepared as described previously (Lara et al., 1982; Vichido et al., 1978). GS activity was measured as described by Ferguson \& Sims (1974). NAD-dependent GDH (EC 1.3.1.2) was measured as described by Fincham (1957). NADH-linked glutamate synthase (GOGAT) (EC 1.4.1.14) was measured by following NADH oxidation as described by Hummelt \& Mora (1980a). Enzyme activities are expressed as units (mg protein) ${ }^{-1}$. One unit is the amount of activity that transforms $1 \mu \mathrm{mol}$ substrate $\mathrm{min}^{-1}$.

In the experiments where GOGAT activity was inhibited in vitro, the enzyme was purified as described by Hummelt \& Mora (1980 b), except that the DEAE column was equilibrated with $0.05 \mathrm{M}$-phosphate buffer. A 14fold purified preparation was obtained. The final concentration of the organic acids was $10 \mathrm{mM}$ and the solutions were adjusted to $\mathrm{pH} 8 \cdot 6$ with $1 \mathrm{M}-\mathrm{KOH}$.

Determination of amino acids. Samples for amino acid analysis were prepared by homogenizing cells with $80 \%$ (v/v) ethanol (Hummelt \& Mora, 1980a). The amino acids were separated by using an Aminco amino acid analyser, and after being coupled with orthophthalaldehyde (Sigma) were quantified in an Aminco ratio fluorimeter.

Determination of tricarboxylic acid cycle intermediates. Mycelial or conidial samples were collected on membrane filters (Millipore type HA, $0.45 \mu \mathrm{m}$ ) and washed with distilled water. Organic acids were extracted by resuspending the samples in $0.6 \mathrm{M}-\mathrm{HClO}_{4}$ and neutralizing with $2.0 \mathrm{M}-\mathrm{K}_{3} \mathrm{PO}_{4}$; they were then determined in the clear supernatant after centrifugation. The extracts were stored at $4{ }^{\circ} \mathrm{C}$ before the determinations were done. 2-Oxoglutarate was determined by measuring the initial rate of change in $A_{340}$ using bovine GDH. A sample of the extract was incubated at room temperature with $30 \mathrm{~mm}$-ammonium sulphate, $0.1 \mathrm{mM}-\mathrm{NADH}$ and $0.01 \mathrm{ml} 2.5 \%$ (v/v) bovine liver GDH [50 units (mg protein) ${ }^{-1}$ ] (Sigma no. G2626) in $1 \mathrm{ml}$ final volume 100 mm-potassium phosphate buffer, $\mathrm{pH} 7 \cdot 6$.

The other tricarboxylic acid cycle intermediates were separated and quantified by HPLC [method of $\mathbf{J}$. Calderón (unpublished)].

Reproducibility of results. The experiments reported were each repeated at least once; representative results are shown.

\section{RESULTS}

\section{Genetic and phenotypic analysis}

The am-132 mutant has a deletion in the NADP-dependent GDH structural gene (GDH') (Kinsey \& Hung, 1981) and is unable to grow on ammonium plus glycine (Fig. 1) or serine, like other mutant strains which have a point mutation in the structural gene of this enzyme (Fincham, 1950; Brett et al., 1976). The am-132 mutant was used as the parental strain for mutant selection. One of the am-132 glycine-resistant $\left(\mathrm{gly} \mathrm{y}^{r}\right)$ mutants isolated was crossed with the wild-type strain $74 \mathrm{~A}$. One am-132;gly mutant obtained from this cross was backcrossed again with the wild-type strain and the progeny were analysed: $57 \%$ were wild-type, $24 \%$ am132 and $19 \% a m-132 ; g l y^{r}$. These results indicated that the $g l y^{r}$ mutation segregated as a monogenic mutation. The work we report here was done with a $\mathrm{GDH}^{-} a m-132 ; g l y^{r}$ double mutant obtained from the latter cross.

Fig. 1 shows the growth of the $a m-132 ; g l y^{r}$ mutant on ammonium plus glycine compared to the am-132 single mutant, which is unable to grow on this nitrogen source (Fincham, 1950), and the wild-type strain, which had a higher growth rate. Unexpectedly, the growth rate of the am$132 ; g l y^{r}$ mutant on ammonium was higher than that of the GDH ${ }^{-}$strain (Fig. 1), but lower than that of the wild-type strain. This same phenomenon was observed when the cultures were grown at $25^{\circ} \mathrm{C}$ (data not shown), when the growth rate on ammonium of the $\mathrm{GDH}^{-}$mutant strain (am) is considerably lower (Hummelt \& Mora, 1980b) than at $37^{\circ} \mathrm{C}$ (Fig. 1). In addition, this new 




Fig. 1

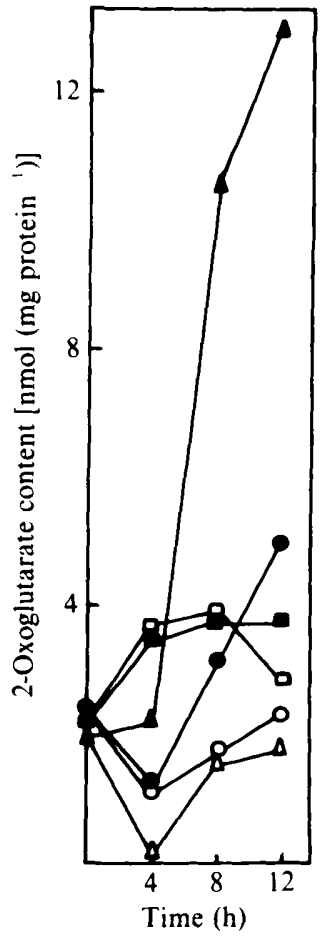

Fig. 2

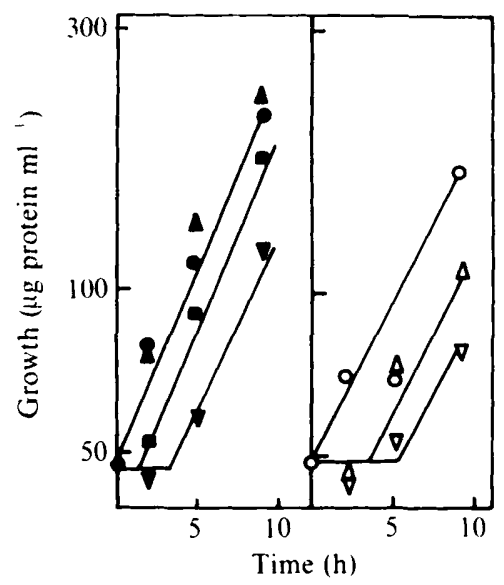

Fig. 3

Fig. 1. Growth of the $a m-132 ; g l y^{r}(O, O)$ and $a m-132(\Delta, \triangle)$ mutants and the wild-type strain $(\square, \square)$ of $N$. crassa on ammonium $(O, \Delta, \square)$ or ammonium $+50 \mathrm{~mm}$-glycine $(O, \triangle, \square)$. The experiment was done four times; results from a representative experiment are shown.

Fig. 2. Intracellular content of 2-oxoglutarate of the $a m-132 ; g l y^{r}(0,0)$ and $a m-132(\Delta, \triangle)$ mutants and the wild-type strain $(\square, \square)$ of $N$. crassa grown on ammonium $(\odot, \Delta, \square)$ or ammonium $+50 \mathrm{~mm}$ glycine $(O, \triangle, \square)$. The experiment was done three times; representative results are shown.

Fig. 3. Inhibition in vivo of growth of the am-132;gly and am-132 mutants by MSF. Conidia were pregerminated for $10 \mathrm{~h}$ in minimal medium at $37^{\circ} \mathrm{C}$ with agitation; MSF was then added to each culture. $, \square, \triangle, \nabla, a m-132 ; g l y^{r}$ mutant $; \bigcirc, \triangle, \nabla, a m-132$ mutant. $O, O$, Minimal medium without MSF; $\triangle, \triangle, 0.01 \mathrm{mM-MSF} ; \square, 0.05 \mathrm{~mm}-\mathrm{MSF} ; \nabla, \nabla, 0.1 \mathrm{mM}-\mathrm{MSF}$. The experiment was done twice; representative results are shown.

mutation also conferred resistance to serine and to methionine sulphoximine (MS) (data not shown), a specific GS inhibitor (Ronzio \& Meister, 1968).

Activities of ammonium-assimilation enzymes in the am-132;gly ${ }^{r}$ mutant

We reported previously the isolation of a glycine-sensitive mutant that maps in the structural gene of GS and has an altered and inactive $\beta$ polypeptide in its GS (Hernández et al., 1986). However, the am-132;gly mutant has a wild-type GS as indicated by the $M_{r}$ and isoelectric point of its $\alpha$ and $\beta$ GS monomers (data not shown). As mentioned before, the $g y^{r}$ mutation segregated independently from the am-132 mutation, which indicates that this new mutation did not map in the structural gene of GS since the $a m$ and $g l n$ loci both map in the VR chromosome and are closely linked (Sober, 1970).

GS activity was not considerably increased in the am-132;gly mutant compared to the am132 strain, either when grown on ammonium or on ammonium plus glycine (Table 1). High GS activity was not responsible for growth on glycine as the am-132 mutant had higher GS activity (Table 1) and was unable to grow on ammonium plus glycine (Fig. 1). The in vitro activity of the GS from the am-132; $g l y^{r}$ mutant was inhibited by glycine in a similar manner to the GS from the am-132 mutant (data not shown). 
Table 1. Enzyme activities in the am-132 and am-132;gly mutants

Enzyme activities (units) were determined after $12 \mathrm{~h}$ incubation on the nitrogen sources indicated. The experiment was done twice; representative results are shown.

\begin{tabular}{clccc} 
Strain & \multicolumn{1}{c}{ Nitrogen source } & GS $^{*}$ & GOGAT & NAD-dependent GDH \\
am-132 & Ammonium & 0.029 & 0.008 & 0.001 \\
& Ammonium + 50 mM-glycine & 0.116 & 0.004 & 0.001 \\
am-132;gly & Ammonium & 0.032 & 0.010 & 0.002 \\
& Ammonium + 50 mM-glycine & 0.052 & 0.009 & 0.001
\end{tabular}

* GS activity was determined by the synthetase assay.

Table 2. Inhibition of GOGAT activity in vitro by MSF in the am-132;gly and am-132 mutants

The experiment was repeated twice; representative results are shown.

$\begin{array}{ccc}\text { MSF concn (mM) } & \overbrace{a m-132}^{\text {Percentage inhibition }} \text { am } 132 \text {;gly } \\ 0.05 & 60 & 67 \\ 0.20 & 74 & 80 \\ 0.40 & 100 & 100\end{array}$

The residual growth of the am-132 mutant or other am mutants on ammonium is due to the presence of GOGAT (Hummelt \& Mora, 1980a,b). It has also been shown that GOGAT does not completely replace NADP-dependent GDH in cultures with an excess of ammonium as the nitrogen source. (Hummelt \& Mora, 1980a,b).

The GOGAT activity of the $a m-132 ; g l y^{r}$ mutant was similar to that of the am-132 mutant (Table 1). So, glycine-resistance cannot be explained by higher induction of GOGAT thus compensating for GS inhibition by glycine, which depends on glutamate concentration (Hernández et al., 1986).

Methionine sulphone (MSF) is a very potent inhibitor of GOGAT from Saccharomyces cerevisiae (Masters \& Meister, 1982). We found that $0.4 \mathrm{~mm}$-MSF completely inhibited GOGAT activity in a dialysed cell extract of the am-132 mutant grown on ammonium (Table 2). GOGAT from the am-132;gly mutant was also inhibited in vitro by MSF (Table 2), which suggests that the glycine-resistance mutation does not result in a structural alteration of GOGAT.

A Saccharomyces cerevisiae mutant that lacks biosynthetic NADP-dependent GDH activity was reported to regain its ability to grow optimally on ammonium when its catabolic NADdependent GDH was induced to very high levels (Grenson et al., 1974). This was not the case for the $N$. crassa glycine-resistant mutant since the catabolic NAD-dependent GDH activity was very low in the am-132;gly mutant as well as in the am-132 strain (Table 1).

\section{Metabolic analysis of the am-132;gly mutant}

If the $g l y^{r}$ mutation could have an effect on the utilization and/or flow of the carbon source, this could result in increased glutamate synthesis. The $\mathrm{GDH}^{-}$mutant did not accumulate 2oxoglutarate when incubated with ammonium plus glycine (Fig. 2) and the 2-oxoglutarate pools of the am-132;gly double mutant grown on this nitrogen source were slightly but significantly higher (Fig. 2). In the am-132 mutant the pyruvate and succinate contents were higher on ammonium than on ammonium plus glycine as the nitrogen source (Table 3 ). In the am-132;gly double mutant the intracellular pools of pyruvate and succinate were similar in the two growth conditions, and they were lower than in the am-132 single mutant (Table 3). 


\section{Table 3. Metabolite contents of the am-132 and am-132;gly mutants}

Metabolite contents [ $\mu \mathrm{mol}$ (mg protein) ${ }^{-1}$ ] were determined after $12 \mathrm{~h}$ incubation on the nitrogen sources indicated. The experiment was done twice; representative results are shown. ND, Not detected.

$\begin{array}{llcccccc}\text { Strain } & \text { Nitrogen source } & \text { Pyruvate } & \text { Succinate } & \text { Glycine } & \text { Glutamate } & \text { Glutamine } & \text { Alanine } \\ \text { am-132 } & \text { Ammonium } & 0.377 & 1.338 & 0.036 & 0.092 & 0.235 & 0.154 \\ & \text { Ammonium + } & 0.219 & 0.881 & 2.570 & \text { ND } & 0.029 & 0.001 \\ & \text { 50 mM-glycine } & & & & & & \\ \text { am-132;glyr } & \text { Ammonium } & 0.157 & 0.593 & 0.056 & 0.057 & 0.185 & 0.093 \\ & \begin{array}{c}\text { Ammonium + } \\ \text { 50 mM-glycine }\end{array} & 0.134 & 0.632 & 1.015 & 0.005 & 0.112 & 0.007\end{array}$

We conclude that the am-132 mutant accumulated several tricarboxylic acid cycle intermediates due to the blockage in glutamate synthesis by NADP-dependent GDH; in the presence of glycine this accumulation did not occur (Fig. 2; Table 3), which may indicate that the carbon flow stops in the latter condition. In the $a m-132 ; g l y^{r}$ double mutant grown on ammonium, there was a low content of tricarboxylic acid cycle intermediates (Fig. 2; Table 3), which suggests a decrease in carbon flow; when glycine was added the lower content of tricarboxylic acid cycle intermediates (Fig. 2; Table 3) suggested that the carbon flow was only slightly decreased as compared to the former condition. The contents of 2-oxoglutarate (Fig. 2), and pyruvate and succinate (Table 3 ), in the am-132;glyr double mutant were closely similar to those of the wild-type strain (Fig. 2). The glycine-resistance mutation was not related to the transport of glycine into the cell since glycine ws accumulated by the am-132;glyr mutant grown on ammonium plus glycine (Table 3 ).

A correlation between the content of tricarboxylic acid cycle intermediates and amino acids was observed as expected. The am- 132 mutant contained no detectable glutamate and had a very low alanine and glutamate content on ammonium plus glycine (Table 3 ) where its growth was abolished (Fig. 1), while the $a m-132 ; g l y^{r}$ double mutant had a detectable glutamate pool (Table 3 ) and its content of alanine and glutamine increased several-fold when grown thus.

From these data we conclude that the am-132;gly mutant synthesizes more glutamate, alanine and glutamine, which is related to its higher capacity to grow on ammonium with or without glycine, and perhaps to a reduced carbon flow.

Curiously, the amino acid pools were higher in the am-132 mutant than in the am-132;gly double mutant when grown on ammonium alone (Table 3). However, the data shown in Table 3 represent the total metabolic pools accumulated after $12 \mathrm{~h}$ incubation on the media indicated, and not the nitrogen and carbon flows of this mutant strain, a result which is discussed below.

\section{Regulation of GOGAT activity by organic acids}

From the experimental data shown, it appears that due to the blockage in the am-132 mutant the intracellular content of several tricarboxylic acid cycle intermediates increases, and this, in turn, lowers the capacity of this mutant to synthesize glutamate. We tested if the latter effect could be due to the inhibition of GOGAT by organic acids. We determined the percentage inhibition of GOGAT activity in a partial purified enzyme preparation of the am-132 mutant grown on ammonium in the presence of different concentrations of several organic acids that are tricarboxylic acid cycle intermediates, or the oxoacids of glycine or serine (Table 4). Glycine did not inhibit $N$. crassa GOGAT activity in vitro (data not shown).

GOGAT from the $a m-132 ; g l y^{r}$ mutant was inhibited by organic acids in a similar manner to the GOGAT from the am-132 mutant (data not shown). This supports our proposition that the glycine-resistance mutation is not due to a structural mutation in GOGAT, which could give a more resistant enzyme. Glycine resistance could rather be related to an increased capacity of GOGAT to synthesize glutamate in vivo in the am-132;gly mutant due to a lower concentration of tricarboxylic acid cycle intermediates (Fig. 2; Table 3) that are inhibitors of GOGAT activity (Table 4). 
Table 4. Inhibition of GOGAT activity by organic acids in the am-132 mutant

\begin{abstract}
Mycelium was grown for $24 \mathrm{~h}$ on minimal medium; GOGAT was partially purified as described in Methods. The concentration of organic acids was $10 \mathrm{mM}$; the $\mathrm{pH}$ was adjusted to 8.6 . The experiment was done twice; representative results are shown.
\end{abstract}

\begin{tabular}{lccc}
\multicolumn{1}{c}{ Organic acid } & 2-Oxoglutarate concn ... & $0.05 \mathrm{mM}$ & $0.1 \mathrm{mM}$ \\
None & & 0 & 0 \\
Succinate & 25 & 19 \\
Malate & 67 & 40 \\
Citrate & 25 & 20 \\
Isocitrate & 40 & 20 \\
Fumarate & 51 & 31 \\
Pyruvate & 25 & 19 \\
Glyoxalate & 40 & 19 \\
$\beta$-Hydroxypyruvate & 36 & 31
\end{tabular}

This postulated increased capacity of GOGAT to synthesize glutamate in vivo in the am$132 ; g l y^{r}$ mutant was investigated by determining its capacity to grow on ammonium plus MSF, compared with the am-132 single mutant. Pregerminated conidia of the am-132;gly mutant had a higher growth rate on ammonium plus $0 \cdot 1 \mathrm{mM}-\mathrm{MSF}$ than pregerminated conidia of the am-132 mutant under the same conditions (Fig. 3). A higher growth rate on ammonium plus MSF was observed in the am-132;glyr mutant, which has lower concentration of tricarboxylic acid cycle intermediates than the am-132 mutant.

We thus conclude that in the am-132;gly mutant there is higher GOGAT activity in vivo than there is in the am-132 mutant, due to the lower concentration in the double-mutant of organic acids (Fig. 2; Table 3) that are GOGAT inhibitors (Table 4). As a result of this increased capacity to synthesize glutamate (Table 3 ) the glycine-resistant mutant is able to grow better than the parental strain on ammonium plus a GS inhibitor such as glycine (Fig. 1), serine or MS and on ammonium plus the GOGAT inhibitor MSF (Table 2; Fig. 3).

\title{
DISCUSSION
}

Fincham (1950) reported that the amino acids glycine or serine completely inhibit growth of am strains on ammonium. We have demonstrated that the effect of glycine and serine is due to inhibition of GS activity (Hernández et al., 1986). It proved possible to obtain a glycine-sensitive strain from wild-type $N$. crassa by a mutation that inactivates the highly active $\beta$ polypeptide of GS (Hernández et al., 1986).

When growth on glutamate of a partial glutamine auxotroph was inhibited by glycine, it was reported that synthesis of glutamate in vivo decreased 100 -fold, even though the content of glutamine only decreased by half; this suggested that the synthesis of glutamine rather than the absolute content of this amino acid, played an important role in optimum cell growth (Hernández et al., 1986). As a result of GS inhibition by glycine, or MS, the carbon flow decreases, and thus growth is prevented due to the double effect of lowering glutamine synthesis and carbon flow (Hernández \& Mora, 1986).

In this paper we reported the isolation of a glycine-resistant mutant from the $\mathrm{GDH}^{-}$strain $a m-132$ that has a deletion in the structural gene for NADP-dependent GDH (Kinsey \& Hung, 1981). This mutation appears to result in diminished carbon flow that cannot be decreased further by the presence of glycine; in the $a m-132 ; g l y^{r}$ double mutant this results in a lower content of tricarboxylic acid cycle intermediates (Fig. 2; Table 3). This is different from what occurs in the $\mathrm{GDH}^{-}$strain am-132, where the carbon flow was almost abolished and GS was totally inhibited in the presence of glycine, as shown by the decrease in glutamate, glutamine, alanine, pyruvate, succinate (Table 3) and 2-oxoglutarate contents (Fig. 2). Although in the 
am-132 strain incubated with ammonium plus glycine, the 2-oxoglutarate content was more drastically reduced (Fig. 2) as compared to the pyruvate and succinate contents (Table 3), even these values represent reduced levels, since they were obtained after $12 \mathrm{~h}$ incubation in the absence of growth.

GOGAT from $N$. crassa was inhibited in vitro by different tricarboxylic acid cycle intermediates and also by the oxoacids of glycine and serine (Table 4). It has been reported that tricarboxylic acid cycle intermediates also regulate GOGAT from $E$. coli (Miller \& Stadman, 1972). West et al. (1967) and Ashby et al. (1974) reported that $N$. crassa NADP-dependent GDH is activated cooperatively by several non-substrate tricarboxylic acid cycle intermediates.

The principal function of GOGAT could be related to the interconversion of glutamine to glutamate and to glutamate recycling (Calderón \& Mora, 1985). This proposal is supported by the fact that GOGAT cannot completely replace NADP-dependent GDH in a GDH ${ }^{-}$mutant strain that has a decreased growth rate on ammonium (Fig. 1), where a low carbon flow and an accumulation of carbon skeletons is observed (Fig. 2; Table 3).

Taking into account that some organic acids were effective inhibitors of GOGAT activity (Table 4), a lower content of these tricarboxylic acid cycle intermediates in the am-132;gly mutant would allow a higher glutamate content (Table 3 ). This increased glutamate content could overcome GS inhibition by glycine, which depends on glutamate concentration (Hernández et al., 1986). Another observation that supports the increased capacity of GOGAT to synthesize glutamate in vivo in the am-132;glyr mutants is that this strain had a higher growth rate on ammonium plus MSF, a GOGAT inhibitor, than the am-132 mutant (Fig. 3).

The ability of the am-132;glyr mutant to grow in the presence of glycine, serine or MS added individually to ammonium as the nitrogen source indicates that resistance is the result of a common effect of glycine, serine and MS. These three GS inhibitors had similar effects on glutamine synthesis and on sucrose catabolism, as shown here and by Hernández \& Mora (1986).

We propose that in the $a m-132 ; g l y^{r}$ mutant an increased carbon flow compared to that of the am-132 mutant elicits increased glutamate and glutamine synthesis that is responsible for an increased growth rate on ammonium (Fig. 1). This proposal can also explain the capacity of the am-132;gly mutant to grow in the presence of glycine, serine, MS or MSF.

By the same token, the slow growth of the am-132 mutant on ammonium as the sole nitrogen source (Fig. 1) is due to the accumulation of tricarboxylic acid cycle intermediates (Fig. 2; Table 3 ); this accumulation results in an inhibition of GOGAT that limits glutamine synthesis, which then reduces the carbon flow (Table 3). This proposal is supported by the accumulation of glutamate and other amino acids (Table 3), observed in the am-132 mutant after $12 \mathrm{~h}$ slow growth on ammonium (Fig. 1); we assume that this limited growth is due to a reduced carbon flow. During the first hours of growth on ammonium, glutamate and glutamine pools were lower in the am-132 mutant than in the am-132;gly mutant (data not shown).

It can be speculated that a glycolytic enzyme less sensitive to ATP inhibition may explain the glyr phenotype.

We are grateful to Jorge Calderón and Lorenzo Segovia for critically reviewing the manuscript, to David Romero for discussion throughout the work and to Maria Elena Velázquez for technical assistance. This work was supported in part by a grant from Consejo Nacional de Ciencia y Tecnologia (CONACyT), México.

\section{REFERENCES}

AshbY, B., WoOtTon, J. C. \& Fincham, J. R. S. (1974). Biochemical Journal 143, 317-329.

Brett, M., Chambers, G. K., Holder, A. A., FinchaM, J. R. S. \& Wootton, J. C. (1976). Mutational amino acid replacements in Neurospora crassa NADP-specific glutamate dehydrogenase. Journal of Molecular Biology 106, 1-22.

Calderón, J. \& Mora, J. (1985). Glutamine cycling in Neurospora crassa. Journal of General Microbiology 131, 3237-3242.
Davis, R. H. \& De Serres, F. J. (1970). Genetic and microbiological research techniques for Neurospora crassa. Methods in Enzymology 17, 79-143.

Ferguson, A. R. \& Sims, A. P. (1974). The regulation of glutamine metabolism in Candida utilis: the role of glutamine in the control of glutamine synthetase. Journal of General Microbiology 80, 159-171.

Fincham, J. R. S. (1950). Mutant strain of Neurospora deficient in aminating ability. Journal of Biological Chemistry 181, 61-73. 
FINCHAM, J. R. S. (1957). A modified glutamic acid dehydrogenase as a result of gene mutation in Neurospora crassa. Biochemical Journal 65, 721-723.

Grenson, M., Dubois, E., Piotrowska, M., DrilLIEN, R. \& AIGLE, M. (1974). Ammonium assimilation in Saccharomyces cerevisiae as mediated by two glutamate dehydrogenases. Molecular and General Genetics 128, 73-85.

HeRnÁNDEZ, G. \& MORA, J. (1986). Glutamine synthesis regulates sucrose catabolism in Neurospora crassa. Journal of General Microbiology 132, 33153323.

Hernández, G., Sánchez-Pescador, R., Palacios, R. \& MoRA, J. (1983). Nitrogen source regulates glutamate-dehydrogenase NADP synthesis in Neurospora crassa. Journal of Bacteriology 154, 524-528.

Hernández, G., MoRA, Y \& MORA, J. (1986). Regulation of glutamine synthesis by glycine and serine in Neurospora crassa. Journal of Bacteriology 165, 133-138.

Hummelt, G. \& MORA, J. (1980a). NADH-dependent glutamate synthase and nitrogen metabolism in Neurospora crassa. Biochemical and Biophysical Research Communications 92, 127-133.

Hummelt, G. \& MORA, J. (1980 b). Regulation and function of glutamate synthase in Neurospora crassa. Biochemical and Biophysical Research Communications 96, 1688-1694.

Kinsey, J. A. \& Hung, B. S. T. (1981). Mutation at the am locus of Neurospora crassa. Genetics 99, 405-414. lara, M., Blanco, L., Campomanes, M., Calva, E., Palacios, R. \& MoRA, J. (1982). Physiology of ammonium assimilation in Neurospora crassa. Journal of Bacteriology 150, 105-112.

MASTERS, D. S., JR \& MEISTER, A. (1982). Inhibition by homocysteine sulfonamide of glutamate synthase purified from Saccharomyces cerevisiae. Journal of Biological Chemistry 257, 8711-8715.

Miller, R. E. \& Stadman, E. R. (1972). Glutamate synthase from Escherichia coli: an iron-sulfide flavoprotein. Journal of Biological Chemistry 247, 7407-7419.

RonZio, R. \& Meister, A. (1968). Phosphorylation of methionine sulfoximine by glutamine synthetase. Proceedings of the National Academy of Sciences of the United States of America 59, 164-170.

SOBER, H. A. (1970). (ed.). Selected data for molecular biology. In Handbook of Biochemistry, pp. I68-69. Cleveland, Ohio: Chemical Rubber Co.

Vichido, I., Mora, Y., Quinto, C., Palacios, R. \& MORA, J. (1978). Nitrogen regulation of glutamine synthetase in Neurospora crassa. Journal of General Microbiology 106, 251-259.

VoGEL, H. J. (1964). Distribution of lysine pathways among fungi: evolutionary implications. American Naturalist 98, 435-446.

West, D. J., TuVeson, R. W., Barrat, R. W. \& FInCHAM, J. R. S. (1967). Allosteric effects in nicotamide adenine dinucleotide phosphate-specific glutamate dehydrogenase from Neurospora crassa. Journal of Biological Chemistry 242, 2134-2138. 